\title{
L-Methionine associated with Hibiscus sabdariffa and Boswellia serrata extracts are not inferior to antibiotic treatment for symptoms relief in patients affected by recurrent uncomplicated urinary tract infections: Focus on antibiotic-sparing approach
}

\author{
Tommaso Cai ${ }^{1}$, Andrea Cocci ${ }^{2}$, Daniele Tiscione ${ }^{1}$, Marco Puglisi ${ }^{1}$, Fabrizio Di Maida ${ }^{2}$, \\ Gianni Malossini ${ }^{1}$, Paolo Verze ${ }^{3}$, Alessandro Palmieri ${ }^{3}$, Vincenzo Mirone ${ }^{3}$, Truls E. Bjerklund Johansen ${ }^{4}$ \\ ${ }^{1}$ Department of Urology, Santa Chiara Regional Hospital, Trento, Italy; \\ ${ }^{2}$ Department of Urology, University of Florence, Florence, Italy; \\ ${ }^{3}$ Department of Urology, University of Naples, Federico II, Naples, Italy; \\ ${ }^{4}$ Department of Urology, Oslo University Hospital and Institute of Clinical Medicine, University of Oslo, Oslo, Norway.
}

\begin{abstract}
Summary Objective: To evaluate the efficacy of a phytotherapic combination of L-Methionine associated with Hibiscus sabdariffa and Boswellia serrata for treatment of acute episodes of uncomplicated urinary tract infections (UTI) in women affected by recurrent UTIs.

Materials and methods: In this randomized phase III clinical trial, adult females with uncomplicated UTI were enrolled into one of the following treatment groups: Group A: phytotherapic combination 1 tablet in the morning and 1 tablet in the evening for 7 days; Group B: Short term antibiotic treatment according to international guidelines recommendations. At baseline, all patients were evaluated by a urologist and quality of life ( $(\mathrm{oL})$ questionnaires and mid-stream urine culture. Same clinical and laboratory investigations were repeated at each follow-up visit. Results: Forty-six patients were enrolled in Group A and 47 in Group B. At the first follow-up (30 days), both groups showed a statistically significant improvement in quality of life scores as compared with baseline assessment [Group A: (2oL $94.3 \mathrm{vs}$ 98.5 p < 0.001); Group B: (QoL 94.5 vs 98.7 p < 0.001)]. An improvement from baseline was also seen at the second followup evaluation after 3 months [Group A: (2oL 94.3 vs $99.1 p<$ 0.001); Group B: (2oL 94.5 vs $98.1 p<0.001)]$. At the second follow-up visit, a statistically significant difference in $Q 0 L$ was reported between the two groups $(99.1$ vs $98.1 ; p<0.003)$ and a transition from UTI to asymptomatic bacteriuria (ABU) was observed 12 of $46(26 \%)$ patients in Group A, while no patients in Group B demonstrated $A B U(p=0.007)$.

Conclusions: Here, we demonstrated that this phytotherapic combination is able, in comparison to antibiotic treatment, to improve patients quality of life, reducing symptoms in acute setting and preventing the recurrences. Interestingly, a significantly higher proportion of patients in the phytotherapy group had ABU after three months. Our findings are of great interest in an antibiotic stewardship perspective.
\end{abstract}

KEY WORDS: Urinary tract infection; L-Methionine; Hibiscus sabdariffa; Boswellia serrata; Plant extracts; Antibiotic stewardship; Treatment.

Submitted 19 March 2018; Accepted 25 March 2018

\section{INTRODUCTION}

Urinary tract infections (UTIs) represent one of the most common community-acquired infectious diseases with significant impact on patients' quality of life and public health care costs (1). More than half of women report having had at least one UTI during their lifetime, and almost $11 \%$ of women suffer from a UTI every year (23). In particular, $20-30 \%$ of women experience another UTI within 6 months after their first episode (1).

The most common management of recurrent UTIs is a short course of oral antimicrobial therapy, with possible alteration of the normal microbial flora of the vagina and/or gastrointestinal tract. Moreover, frequent use of antibiotics for treatment and prevention of UTIs increases healthcare costs and the spread of multidrug-resistant microorganisms (4-7). Nowadays, there are no universally accepted recommendations on prophylactic antibiotic management to prevent recurrent UTI (3). Reduced antibiotic prescription would not only prevent resistance but will also lower public health care costs. In this scenario, the use of phytotherapy for symptom relief and decrease of symptomatic recurrences in UTI is an intriguing alternative (3). The aim of the present study was to compare the effectiveness of a combination of L-Metionina associated with Hibiscus sabdariffa and Boswellia serrata (Acidif plus ${ }^{\circledR}$ ) and standard antibiotic treatment of acute episodes of uncomplicated UTI in women affected by recurrent UTI, focusing on QoL improvement and the capability to reduce antibiotic usage.

\section{Materials AND MEthods}

Study design

This is a single Centre, randomized and controlled phase III study. From January to June 2017, we enrolled all women affected by recurrent uncomplicated UTI seen in our referral centre. Written informed consent was obtained from all subjects before proceeding with the enrollment. Baseline assessment comprised evaluation by urologist, completion of QoL questionnaires and a mid-stream urine culture. All patients in the antibiotic arm were treated according to an empirical approach, in line with the 
European Association of Urology (EAU) guidelines recommendations (8). The results of mid-stream urine culture were used only for confirmation of the diagnosis and not for tailoring the treatment. All selected patients were, then, randomly allocated into one of the two following groups:

- Group A: one-week therapy of Acidif Plus ${ }^{\circledR}, 1$ tablet in the morning and 1 tablet in the evening for 7 days.

- Group B: Short term antibiotic therapy according to the trialist's choice based on the EAU guidelines on urological infections (8).

After one week from the start of treatment, all patients were contacted by phone in order to ensure compliance to the treatment in both arms. The follow up schedule included urologic visit after one and three months with QoL questionnaires and mid-stream urine cultures.

The study schedule is shown in Figure 1. underwent a short course of antibiotic therapy according to the antibiogram and guidelines recommendations.

\section{Outcome measures and statistical analysis}

The primary endpoint was the rate of responders defined as the demonstration of urinary pathogens $<103$ $\mathrm{CFU} / \mathrm{mL}$ in a mid-stream urine culture on the second and third follow up visit (after one and three months respectively from the beginning of the treatment) as well as improvement in symptoms scores. Data about the compliance to treatment, frequency of UTI recurrences or transition from symptomatic UTI to asymptomatic bacteriuria at subsequent follow up visits were also recorded. In order to obtain valid results for analysis, sample size calculation was based on the following assumptions. Difference between the groups, $3 \pm 1$ score

\section{Figure 1.}

The figure shows the study schedule. QoL: quality of life evaluation. Group A: one-week therapy of ACIDIF PLUS ${ }^{\circledR}$, 1 tablet in the morning and 1 tablet in the evening for 7 days. Group B: Short term antibiotic therapy according to the trialist's choice and EAU guidelines on urological infections.

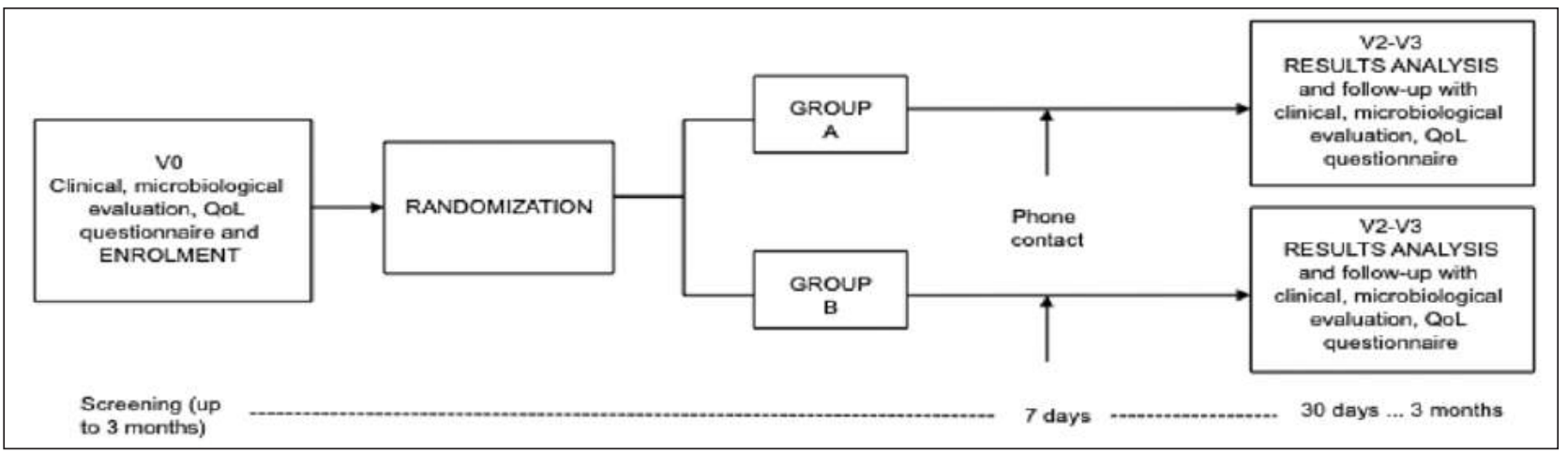

\section{Composition and characterization}

of the phytotherapeutic extracts used

All patients who were randomized to the Acidif Plus ${ }^{\circledR}$ group received oral administration of 1 tablet of Acidif Plus ${ }^{\circledR}$ in the morning and 1 tablet in the evening, in line with the manufacturer's instructions (Biohealth srl, Rivoli - TO), Italy (http://www.biohealth.it/it/prodotti/linea-acidif/25-acidif-plus). Each dose contained a combination of $400 \mathrm{mg}$ of LMethionine associated with $100 \mathrm{mg}$ of Hibiscus sabdariffa and $100 \mathrm{mg}$ of Boswellia serrata (L.) Roxb.

\section{Inclusion and exclusion criteria}

Exclusion criteria comprised: evidence of overactive bladder; anatomical abnormalities or previous surgery of the urinary tract; complicated UTI; intake of antibiotics within 4 weeks before enrollment; pregnancy; indwelling urinary catheter or stent.

\section{Microbiological and clinical considerations}

Treatment failure in group A was defined as microbiological colonization of at least 105 colony-forming units per milliliter (CFUs/mL) of a single uropathogen in the midstream urine culture associated with the persistence of urinary tract symptoms. Transition from symptomatic UTI to asymptomatic bacteriuria was not considered as treatment failure, but was registered as an improvement in symptoms. In case of treatment failure in group $\mathrm{A}$, patients points in the QoL questionnaire; $\alpha$ error level, 0.05 twosided; statistical power, 80\%; anticipated effect size, Cohen's $\mathrm{d}=0.5$. The calculation yielded $2 \times 41$ individuals per group. Taking into account a drop-out rate of $10 \%$, the final sample size was set to 90 patients in both groups. Statistical analysis was performed by using SPSS.

\section{RESULTS}

Ninety-three female patients with clinical evidence of uncomplicated UTI entered the study. Forty-six and fortyseven patients were enrolled in Group A (mean age 46.5 range: 23-62) and Group B (mean age 47.1 range: 22-63), respectively. Demographic and clinical characteristics of the study population are shown in Table 1.

The most commonly isolated bacterial strain at the time of enrollment was Escherichia Coli (E. coli) in 86.9\% (40/46) of patients in Group A and in 82.9\% (39/47) in Group B, respectively.

\section{Treatment compliance and treatment related adverse effects}

After 7 days treatment compliance was complete in Group A (100\%), while within Group B 14.9\% (7/47) of patients discontinued the prescribed therapy because of drug-related adverse effects. No side effects occurred in the phytotherapy group. 
Table 1.

Demographic, clinical and microbiological characteristics of all enrolled and analysed women, at the baseline.

Group A: one-week therapy of Acidif Plus ${ }^{\circledR}, 1$ tablet in the morning and 1 tablet in the evening for 7 days.

Group B: Short term antibiotic therapy according to the trialist's choice and EAU guidelines on urological infections.

\begin{tabular}{|c|c|c|c|}
\hline \multicolumn{4}{|c|}{ Demographic and clinical characteristics of the study population at baselin } \\
\hline & Group A & Group B & $\mathbf{P}$ \\
\hline Enrolled patients & 46 & 47 & \\
\hline Age (mean) & 46.5 & 47.1 & \\
\hline Previous UTI per year (mean) & 4.3 & 4.7 & \\
\hline $\begin{array}{l}\text { Number of sexual partners } \\
1 \\
>1\end{array}$ & $44(95.6)$ & $43(91.4)$ & \\
\hline \multicolumn{4}{|l|}{ Marital status } \\
\hline Married & $31(67.3)$ & $29(61.7)$ & \\
\hline Single & $15(32.7)$ & $18(38.3)$ & \\
\hline Sexual encounters per week (mean) & 2.1 & 2.0 & \\
\hline \multicolumn{4}{|l|}{ Bacterial strains } \\
\hline E. coli & $40(86.9)$ & $39(82.9)$ & \\
\hline E. faecalis & $2(4.4)$ & $2(4.3)$ & \\
\hline E. faecium & $1(2.2)$ & $1(2.2)$ & \\
\hline Klebsiella spp. & $3(6.5)$ & $5(10.6)$ & \\
\hline
\end{tabular}

First follow-up evaluation (30 days)

At one month follow up visit, a statistically significant clinical improvement was reported in 95.7\% (44/46) of patients in Group A (QoL 94.3 vs $98.5 \mathrm{p}<0.001)$ and 100\% (47/47) in Group B (QoL 94.5 vs 98.7 p < 0.001), respectively. No statistically significant differences emerged when comparing QoL results in the two groups. A sterile urine culture was reported in $80.4 \%$ (37/46) and in $95.7 \%$ (45/47) of patients in Group A and Group B, respectively. Notably, in the remaining $19.6 \%(9 / 46)$ of

Table 2.

Outcome variables before and after treatment, in the two groups. Group A: one-week therapy of Acidif Plusn ${ }^{\circledR}, 1$ tablet in the morning and 1 tablet in the evening for 7 days. Group B: Short term antibiotic therapy according to the trialist's choice and EAU guidelines on urological infections. $A B U$ : asymptomatic bacteriuria; UTI: symptomatic urinary tract infection; n.s.: not statistically significant.

\begin{tabular}{|c|c|c|c|c|}
\hline Outcomes variable & Baseline & $\begin{array}{l}\text { Follow-up } \\
30 \text { days }\end{array}$ & $\begin{array}{c}\text { Follow-up } \\
90 \text { days }\end{array}$ & $\mathbf{P}$ \\
\hline \multicolumn{5}{|l|}{ Clinical improvement } \\
\hline Group A & $0 / 46$ & $44 / 46(95.5)$ & $44 / 46(95.5)$ & $<0.001$ \\
\hline Group B & $0 / 47$ & $47 / 47(100)$ & $45 / 47(95.7)$ & $<0.001$ \\
\hline$p$ (between the groups) & & n.s. & n.s. & \\
\hline \multicolumn{5}{|l|}{ QoL (questionnaire) } \\
\hline Group A & 94.3 & 98.5 & 99.1 & $<0.001$ \\
\hline Group B & 94.5 & 98.7 & 98.1 & $<0.001$ \\
\hline$p$ (between the groups) & & n.s. & $<0.003$ & \\
\hline \multicolumn{5}{|l|}{$\begin{array}{l}\text { Microbiological improvement } \\
\text { Sterile }\end{array}$} \\
\hline Sterile urine & & & & \\
\hline Group A & $\begin{array}{l}0 / 46 \\
0 / 47\end{array}$ & $\begin{array}{l}37 / 46(80.4) \\
45 / 47(95.7)\end{array}$ & $\begin{array}{l}34 / 46(73.9) \\
44 / 47(93.6)\end{array}$ & $\begin{array}{l}\text { n.s. } \\
\text { n.s. }\end{array}$ \\
\hline p (between the groups) & & n.s. & n.s. & \\
\hline \multicolumn{5}{|l|}{ Transition to ABU from UTI } \\
\hline Group A & - & $9 / 46(19.6)$ & $12 / 46(26)$ & n.s. \\
\hline Group B & - & $2 / 47(4.3)$ & $0 / 46$ & n.s. \\
\hline$p$ (between the groups) & & n.s. & 0.007 & \\
\hline
\end{tabular}

patients within Group A a transition from symptomatic UTI to asymptomatic bacteriuria (ABU) was observed and also a change in microorganism (from $E$. coli to Enterococcus faecalis). On the contrary, only 4.3\% (2/47) of patients among Group B showed a transition from UTI to ABU. No treatment failures were recorded in Group A. All findings at the first follow-up are shown in Table 2.

\section{Second follow-up evaluation (90 days)}

At the 3 month follow up visit, 95.7\% (44/46) of patients in Group A showed a clinically significant improvement (QoL 94.3 vs $99.1 \mathrm{p}<0.001$ ), as well as $95.7 \%(45 / 47)$ patients in Group B (QoL 94.5 vs $98.1 \mathrm{p}<0.001$ ). A statistically significant difference emerged in terms of QoL between the two groups ( $p<0.003 ; 99.1$ vs 98.1)

In Group A, 73.9\% (34/46) of patients demonstrated a second sterile mid stream urine culture, while the remaining $26 \%(12 / 46)$ of patients showed a transition from symptomatic UTI to ABU and a change of microorganism (from $E$. coli to Enterococcus faecalis) $(\mathrm{p}=0.007)$. Again, no treatment failures were recorded in Group A while 2 patients in Group B showed symptomatic UTI with microbiological demonstration of Escherichia coli. No patients in Group B showed transition from UTI to ABU. Table 2 shows all findings at the second follow-up visit.

\section{Discussion}

Although antimicrobials remain the mainstay of treatment for UTI, the spread of multidrug resistant microorganisms among community-acquired isolates is worrying and calls for stronger surveillance and new preventive approaches. Today, inappropriate antibiotic use and antimicrobial resistance have become major issues worldwide. Unfortunately, there is no "gold standard" prophylactic regimen to prevent recurrent UTI. Actually, EAU guidelines present several non-antibiotic measures for preventing recurrent UTIs but only few are based on well-designed randomized clinical trials (8).

Furthermore, many women have signs of UTIs without evidence of bacterial presence, a clinical condition known as $\mathrm{ABU}$. Although $\mathrm{ABU}$ is usually treated in women with recurrent UTIs there is no clear indication for this treatment. A significant body of recent evidence $(6-7,9)$ has demonstrated that antibiotic treatment of ABU is not only pointless, but could even be harmful. Particularly, in a randomized controlled study, Cai et al. showed that after a 12-month period of antimicrobial therapy of $\mathrm{ABU}$ in women affected by recurrent UTIs, the rate of symptomatic UTIs was higher in patients treated with antibiotics. There was also a higher prevalence of multidrug-resistant E. coli after antibiotic treatment of $\mathrm{ABU}$ due to Enterococcus faecalis (9). These data suggest that ABU could play a protective role in preventing symptomatic recurrences, interfering with the settlement in the urinary tract of many enteric pathogens, such as E. coli. In this sense, a non-antibiotic approach should be preferred because it doesn't modify the normal commensal bowel flora. As a result, research on non-antibiotic prophylaxis of recurrent UTIs has gradually grown during recent years. Particularly, the use of phytotherapy and neutraceuticals could represent a feasible alternative 
approach for reducing the use of antibiotics and decreasing the rate of symptomatic recurrences $(3,10-11)$. In this light, we wanted to evaluate an oral supplementation of L-Methionine combined with Hibiscus sabdariffa and Boswellia serrata (named Acudif plus ${ }^{\circledR}$ ). L-Methionine is a sulfur donor which is necessary for the biosynthesis of cysteine, a sulfide aminoacid. A physiological $\mathrm{pH}$ is obtained by the action of L-Methionine which creates unfavorable conditions for bacterial colonization since a large number of Gram-negative bacteria are able to alkalize the urine by enzymatic degradation of urea. Furthermore, dry extract of Hibiscus sabdariffa is a phytocomplex containing Sambubiosides, which seems to have an anti-inflammatory activity by lowering the levels of inflammatory mediators including iNOS, NO, IL-6, MCP1 , and TNF- $\alpha$ induced by LPS (12). Moreover, several preliminary studies demonstrated that Hibiscus sabdariffa is able to prevent recurrent uncomplicated urinary tract infections due to its inhibitory activity against Escherichia coli and Candida albicans (13). Finally, Boswellia serrata also seems to inhibit some inflammation mediators, in particular 5-lipoxygenase, with a subsequent decrease in inflammatory response mediated by leukotrienes (14). In our knowledge, this is the first study evaluating the prophylactic effect of L-Methionine associated with Hibiscus sabdariffa and Boswellia serrata extracts in women suffering from recurrent uncomplicated UTIs. In a recent multicenter observational study Passaro et al. (15) had already assessed the effectiveness of Acidif Plus ${ }^{\circledR}$ in reducing the bacterial load and improving symptoms in pregnant women affected by symptomatic UTI. In our experience, Acid Plus ${ }^{\circledR}$ was well tolerated with optimal compliance and no side effects. As many as $95.7 \%$ (44/46) of patients demonstrated relevant clinical improvement with a statistically significant difference compared to women treated with standard antibiotic therapy 3 months after the beginning of treatment. Moreover, at first follow up visit almost $20 \%$ of patients within Group A showed a transition from symptomatic UTI to ABU with lasting results at 3 months. Noteworthy, the fact that Acidif Plus ${ }^{\circledR}$ was able to establish an ABU is a tangible proof of absence of effect on the normal urine microbiota. Increased understanding of the action of phytotherapy and nutraceuticals will enable us to develop appropriate non-antibiotic treatment and prevention strategies for recurrent UTIs (16). Larger highquality studies are required to confirm the findings in our study.

\section{Conclusions}

Urinary tract infections affect a large number of women and many suffer from recurrent UTIs. Actually, current antibiotic treatments select multi-drug resistant microorganism, both in the gut and in the bladder, and this practice is no longer sustainable. Use of phytotherapy and nutraceuticals is an intriguing approach to reduce the excessive prescription of antibiotics. In the present study we could demonstrate that Acidif Plus ${ }^{\circledR}$ was able to improve patients QoL, lower symptoms in the acute setting and prevent UTI recurrences in women with recurrent UTIs as compared to antibiotic treatment. Further prospective studies are needed to validate our results.

\section{REFEREnCES}

1. Silverman JA, Schreiber HL $4^{\text {th }}$, Hooton TM, Hultgren SJ. From physiology to pharmacy: developments in the pathogenesis and treatment of recurrent urinary tract infections. Curr Urol Rep. 2013; 14:448-56.

2. Foxman B, Barlow R, D'Arcy $H$, et al. Urinary tract infection: selfreported incidence and associated costs. Ann Epidemiol. 2000; 10:509-515.

3. Cai T, Tamanini I, Kulchavenya E, et al. The role of nutraceuticals and phytotherapy in the management of urinary tract infections: What we need to know? Arch Ital Urol Androl. 2017; 89:1-6.

4. Kostakioti M, Hultgren SJ, Hadjifrangiskou M. Molecular blueprint of uropathogenic Escherichia Coli virulence provides clues toward the development of anti virulence therapeutics. Virulence. 2012; 3:592-594.

5. Gupta K, Scholes D, Stamm WE. Increasing prevalence of antimicrobial resistance among uropathogens causing acute uncomplicated cystitis in women. JAMA 1999; 281:736-8.

6. Cai T, Mazzoli S, Mondaini N, et al. The role of asymptomatic bacteriuria in young women with recurrent urinary tract infections: to treat or not to treat? Clin Infect Dis. 2012; 55:771.

7. Cai T, Koves B, Johansen TE. Asymptomatic bacteriuria, to screen or not to screen - and when to treat? Curr Opin Urol. 2017; 27:107-111.

8. European Association of Urology Guidelines on Urological Infections. http://uroweb.org/guideline/urological-infections/

9. Cai T, Nesi G, Mazzoli S, et al. Asymptomatic bacteriuria treatment is associated with a higher prevalence of antibiotic resistant strains in women with urinary tract infections. Clin Infect Dis. 2015; 61:1655-61.

10. Stange R, Schneider B, Albrecht $U$, et al. Results of a randomized, prospective, double-dummy, double-blind trial to compare efficacy and safety of a herbal combination containing Tropaeoli majoris herba and Armoraciae rusticanae radix with cotrimoxazole in patients with acute and uncomplicated cystitis. Res Rep Urol. 2017; 9:43-50.

11. Ledda A, Belcaro G, Dugall M, et al. Highly standardized cranberry extract supplementation (Anthocran ${ }^{\circledR}$ ) as prophylaxis in young healthy subjects with recurrent urinary tract infections. Eur Rev Med Pharmacol Sci. 2017; 21:389-393.

12. Sogo T, Terahara N, Hisanaga A, et al. Anti-inflammatory activity and molecular mechanism of delphinidin 3-sambubioside, a Hibiscus anthocyanin. Biofactors. 2015; 4:58-65.

13. UTirose ${ }^{\mathrm{TM}}$ : a natural answer to urinary tract infections issues. Brochure produit, RSO -002/1, certifié par Bureau Veritas.

14. Pandey RS, Singh BK, Tripathi YB. Extract of gum resins of Boswellia serrata L. inhibits lipopolysaccharide induced nitric oxide production in rat macrophages along with hypolipidemic property. Indian J Exp Biol. 2005; 43:509-16.

15. Passaro M, Mainini G, Ambrosio F, et al. Effect of a food supplement containing L-Methionine on urinary tract infections in pregnancy: a prospective, multicenter observational study. J Altern Complement Med. 2017; 23:471-478.

16. Cai T, Mazzoli S, Migno S, et al. Development and validation of a nomogram predicting recurrence risk in women with symptomatic urinary tract infection. Int J Urol. 2014; 21:929-34.

\section{Correspondence}

Tommaso Cai, MD - ktommy@libero.it

Daniele Tiscione, MD- Marco Puglisi, MD - Gianni Malossini, MD

Department of Urology, Santa Chiara Hospital

Largo Medaglie d'Oro 9, Trento, Italy

Andrea Cocci, MD - Fabrizio Di Maida, MD

Department of Urology, University of Florence, Florence, Italy

Paolo Verze, MD - Alessandro Palmieri, MD - Vincenzo Mirone, MD Department of Urology, University of Naples, Federico II, Naples (Italy)

Truls E. Bjerklund JohanseN, MD

Department of Urology, Oslo University Hospital and Institute of Clinical Medicine, University of Oslo, Oslo (Norway) 